\title{
Review Article Amatoxin: A Review
}

\section{Brandon Allen, Bobby Desai, and Nate Lisenbee}

Department of Emergency Medicine, University of Florida College of Medicine, 1329 SW 16th Street, P.O. Box 100186, Gainesville, FL 32610-0186, USA

Correspondence should be addressed to Bobby Desai, bdesai@ufl.edu

Received 19 June 2012; Accepted 30 July 2012

Academic Editors: P. Eisenburger and O. Karcioglu

Copyright (c) 2012 Brandon Allen et al. This is an open access article distributed under the Creative Commons Attribution License, which permits unrestricted use, distribution, and reproduction in any medium, provided the original work is properly cited.

This paper presents a comprehensive review of amatoxin poisoning. The paper discusses the biochemistry of amatoxin, as well as the clinical manifestations of amatoxin ingestion. In addition, the evaluation of the patient with amatoxin ingestion is discussed, along with the treatment-including newer therapy—and the ultimate prognosis of the syndrome.

\section{Introduction}

The United States contains over 5,000 species of mushrooms. While $4-6 \%$ of US mushroom species are safe to eat, approximately $2 \%$ are poisonous, with twelve species being known to be fatal if ingested. Worldwide, accurate figures for the incidence of mushroom toxicity are difficult to obtain. Outbreaks of severe mushroom poisoning have been documented in Europe, Russia, the Middle East, and the Far East. In all of these areas, mushroom foraging is a common practice.

Ninety percent of deaths from mushroom ingestions are due to amatoxin, which possesses a fatality rate of up to $25 \%$ when ingested. In 2007, 45 cases of amatoxin poisoning were reported in the United States, with only one documented death. The difficulty in the treatment of amatoxin poisoning lies in part to the lack of both an effective antidote and a standardized accepted treatment for this poisoning. On a molecular level, amatoxin and its effects on downstream physiologic targets is just beginning to be understood, thus leading to the lack of progress in therapeutic options for amatoxin poisoning [1]. The majority of reported amatoxin ingestions fall in a few specific categories, such as accidental ingestions in young children or wild mushroom foragers and intentional ingestions in patients attempting suicide or seeking the drug for its hallucinatory affects [1].

In terms of biochemical categorization, amatoxin exists as a heat-stable toxin, which allows it to maintain its threedimensional structure at elevated temperatures. Increasing amatoxin toxicity is the fact that it is not susceptible to enzymatic hydrolization. Amatoxin exists in multiple subtypes, with alpha and beta being the common, and all subtypes are not water soluble. Through the mechanism of mRNA inhibition, amatoxin is able to inhibit DNA dependent RNA polymerase $B$ which leads to a wide range of tissue injury, most notably to the intestinal mucosa, liver, and kidneys. Enterohepatic circulation followed by renal reabsorption delivers the toxin to these tissues. In terms of toxicity, there are reported cases of amanita poisoning and toxicity with ingestions as small as 30 grams of Amanita phalloides [2].

Many species of mushrooms contain amatoxin. For example, Aminita phalloides, known as the "Death Cap," Amanita verna, known as the "Fool's Mushroom," and Amanita virosa and Amanita bisporigera, a complex known as the "Destroying Angel," all contain alpha-amanitin. Galerina autumnalis, known as the "Autumn Skullcap," contains betaamanitin and gamma-amanitin, which are believed to be less toxic than their alpha-subtype counterpart [3].

It is well-known that amatoxin primarily causes death through the process of fulminant hepatic failure secondary to liver parenchymal necrosis. Zhou et al. created the first acceptable large animal model for fulminant hepatic failure through exposure to intraperitoneal infusions of amatoxin and endotoxin. Liver biopsy revealed extensive hepatocyte steatosis and parenchymal necrosis at as early as 36 hours after amatoxin administration. Extrahepatic manifestations were confirmed in the form of hepatic encephalopathy 


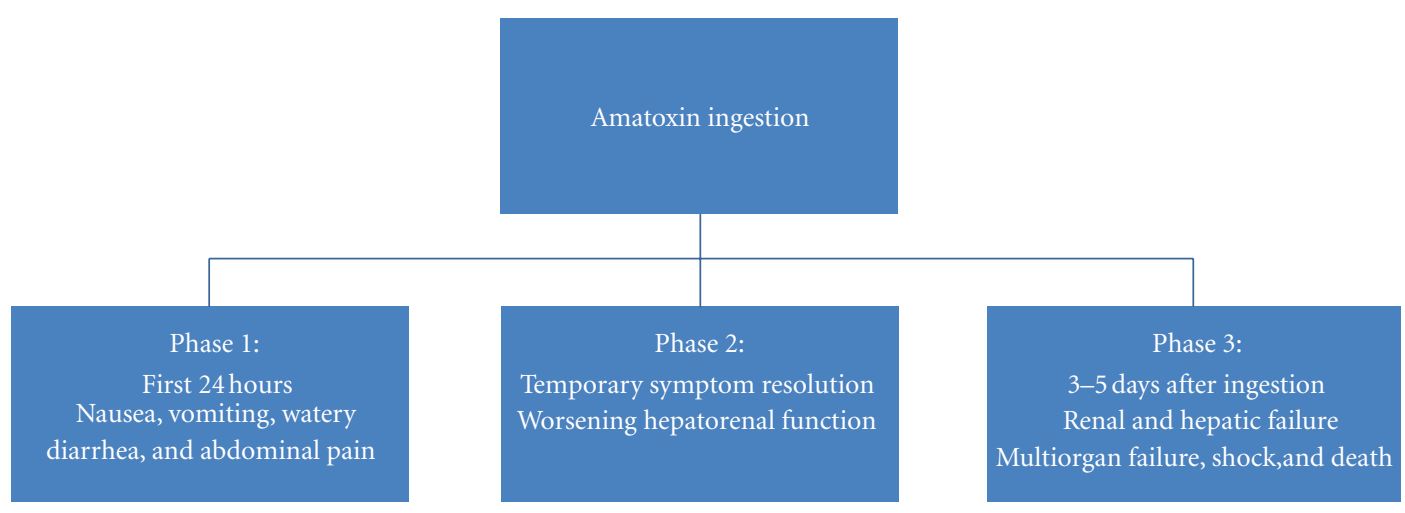

FIgURE 1: Phases of amatoxin ingestion.

as seen on MRI and autopsy. The authors of this study confirmed that fulminant hepatic failure is a rapid and progressive pattern of disease that is important to understand for any suspected amatoxin poisoning [4].

\section{Clinical Presentation}

The pattern of injury following amatoxin ingestion presents in three phases. The first phase consists of ingestion and the appearance of initial symptoms within the first 24 hours, with the majority of patients being symptomatic within the first 15 hours. The constellation of symptoms involved in phase one include nausea, vomiting, watery diarrhea, and abdominal pain, which may be confused for cholera, gastroenteritis, or food poisoning depending on the clinical scenario. In general, gastrointestinal symptoms resolve in the beginning of the second phase, which typically begins around 24 hours after ingestion. As phase two progresses, renal and hepatic dysfunction dominate the clinical picture. The temporary resolution of symptoms early in phase two often leads to premature hospital or emergency department discharge. Within days, this group of patients often present back to the emergency department in hepato-renal failure with clinically evident jaundice. The third and final phase of amatoxin poisoning occurs three to five days after ingestion and consists of further progression of renal and hepatic failure. Without focused care, phase three can lead to shock, multiorgan failure, and death [5]. These patients often will require an emergency liver transplant. This can be seen schematically in Figure 1.

\section{Evaluation}

Laboratory evaluation of patients with suspected alphaamanitin toxicity should be thorough and should at a minimum, include liver function testing, coagulation factors, ammonia, fibrinogen, bilirubin, complete blood count, electrolyte analysis, amylase and lipase, and urinalysis. While amatoxin ingestion is often mistaken for other diagnoses, clinicians are likely to order a large majority of the necessary labwork simply due to the patient's clinical picture. After admission, when confirmatory tests are performed within the first 48 hours, they have been found to be highly sensitive with a high negative predictive value for the detection of alpha-amanitin. Combining clinical manifestations with analysis of the patient's urine for alpha-amanitin has been documented by Butera et al. to improve diagnostic accuracy. In epidemiologic terms, the most precise diagnostic approach is achieved through combining "both the high sensitivity and negative predictive value of the clinical assessment performed by an experienced toxicologist, and the high specificity and positive predictive value that characterize urinary amanitin analysis [6]." However, it is important to note that a negative urinalysis for alpha-amanitin is only dependable as a marker for poisoning if performed within 36 hours of ingestion. Urine can be analyzed by 3 primary methods for the detection of alpha-amanitin as follows: radioimmunoassay (RIA), high-performance liquid chromatography (HPLC), or enzyme-linked immunosorbent assay (ELISA). Unfortunately, the relationship of alphaamanitin levels in the urine does not coincide with the severity of liver damage or the prognosis of the patient. Thus, it cannot be used as a prognostic tool following amatoxin ingestion $[6,7]$.

\section{Therapy/Management}

Treatment successes for amatoxin ingestion have been few and far between; the majority of success has been noted in case reports with little evidence-based data behind the recommendations. Besides liver transplant, the known proposed therapies are primarily supportive.

In the patient with known amatoxin ingestion, there are some time-sensitive therapies that are employed for gastrointestinal (GI) decontamination secondary to the toxin's enterohepatic circulation. Multiple doses of activated charcoal (MDAC) are recommended in the early stages after ingestion as well as gastric lavage (if ingestion less than six hours prior to arrival).

Medical therapies for amatoxin ingestion range from antibiotics to herbal remedies. Of note, milk thistle (Silybum marianum), a therapy known for its hepatoprotective, antiinflammatory, and antioxidant properties, has been used as an emergency antidote to amatoxin poisoning $[8,9]$. Animal 
studies have found that milk thistle extract completely counteracts the toxic effects of the mushroom when given within 10 minutes of ingestion. If given within 24 hours, it significantly reduces the risk of liver damage and death [9].

In 2007, a study was done on mice exposed to amatoxin to assess the efficacy of five theoretical therapies in the prevention of hepatotoxicity. The potential therapies included $\mathrm{N}$-acetylcysteine, benzylpenicillin, cimetidine, thioctic acid, and silybin. Results showed that none of the five therapies when compared to the control groups, significantly reduced the rise in aminotransferases, associated with amatoxin poisoning. Also, histological analysis showed no decrease in hepatic necrosis in the study groups when compared to the control groups. Thus, the researchers concluded that the five analyzed therapies were not effective in preventing hepatotoxicity after amatoxin poisoning [10].

In a 2010 article in Toxicon, Poucheret et al. reported on 2110 patients hospitalized for amatoxin poisoning. In total, the average mortality rate from amatoxin poisoning was $11.58 \%$. In this review, 1632 of the total 2110 received some form of chemotherapeutics. In this subset of patients, 174 patients died, leading to an average mortality rate of $10.66 \%$ when treated with chemotherapy. A total of thirteen different chemotherapeutic agents were used in studied patients, some as single-agent therapies and some as combination therapies [1].

One of the more promising agents used in this study was silybin, which was used both as a single agent and in combination with other agents for therapy. The mortality rate in patients who received silybin - whether solely or in combination with other therapies-was 5.6\%, almost half of the overall mortality rate $(10.66 \%)$ in the treated patient subset. Adding credence to this result is the large total number of patients in the study that received silybin $(624$ patients), which is $38.3 \%$ of the total number of patients treated. In addition to silybin, NAC showed somewhat comparable results by reducing the average mortality to $6.8 \%$; however, the sample size was significantly smaller with NAC [1]. This is in direct contrast to an earlier study, which showed no significant benefit [10].

Benzathine penicillin is one of the most commonly used therapeutic options but demonstrates little efficacy [11]. Mechanism of action is believed to reduce/inhibit liver uptake of amatoxin. The proposed dose is up to 1 million units $/ \mathrm{kg} /$ day IV. Aside from these therapies, the primary recommendations are supportive care if the known time of ingestion is greater than six hours.

A review of a specific therapeutic protocol was published in 2007 assessing this protocol potential effectiveness for the treatment for amatoxin poisoning. In this study, a sevenstep protocol was initiated on patients suspected of having amatoxin poisoning:

(1) intensive supportive therapy with careful correction of water, glucose, electrolyte imbalances, and acidbase status;

(2) correction of the altered coagulation factors, with fresh frozen plasma coupled with the intravenous administration of vitamin $K_{1}$ ( 20 to $40 \mathrm{mg} /$ day) in patients with a PTA of less than $66 \%$ (INR > 2.1);

(3) oral administration of multiple-dose activated charcoal (20 to $40 \mathrm{~g}$ every 4 hours) for at least three days after ingestion;

(4) fluid therapy ( $1 \mathrm{~L}$ crystalloid/10 kg/day) and mannitol $18 \%(0.25$ to $0.5 \mathrm{~g} / \mathrm{kg} / \mathrm{h})$ in order to obtain a moderately enhanced diuresis $(200 \mathrm{~mL} / \mathrm{h})$ for two days after ingestion;

(5) intravenous administration of dexamethasone (8 to $16 \mathrm{mg} /$ day);

(6) intravenous administration of glutathione (4.8 g/day in two divided doses);

(7) continuous intravenous administration of high doses of $\mathrm{Na} / \mathrm{K}$ penicillin $\mathrm{G}(1,000,000 \mathrm{IU} / \mathrm{kg}$ for the first day, then $500,000 \mathrm{IU} / \mathrm{kg}$ for the next two days).

The researchers also noted that metoclopramide was used to treat symptoms of nausea and vomiting in those patients that required it. After analysis of data, the study concluded that all patients treated with this protocol within 36 hours of ingestion fully recovered without lasting negative effects [12].

\section{Prognosis}

Patients that typically die from amatoxin poisoning (up to $25 \%$ of amatoxin ingestion results in a fatal outcome) are more likely to have low-mean arterial pressure, hepatic encephalopathy, and hemorrhage secondary to a decrease in clotting factors. They are also likely to have oliguria and anuria, as acute renal failure is noted in a significant percentage of patients with amatoxin poisoning. Furthermore, they are likely to have hypoglycemia due to lack of glycogen stores and thrombocytopenia. Other values associated with an increased mortality include low sodium, elevated urea, AST, ALT, total bilirubin, prothrombin time, international normalized ratio, and activated partial thromboplastin time. Mortality is almost assured once fulminant hepatic failure has ensued, unless an emergent liver transplant can be arranged. In patients that survive amatoxin poisoning, the most accurate predictors of survival outcome appear to be the trending of hepatic transaminases and coagulation factors.

There are multiple proposed criteria for the indication of emergent liver transplant following amatoxin ingestion; however, the King's College criteria has been shown to be most efficacious [13] as follows:

(1) $\mathrm{PT}>100($ INR $>6.5)$;

(2) 3 of the following:

(a) PT $>50($ INR $>3.5)$

(b) Serum bilirubin $>17.5 \mathrm{mg} / \mathrm{dL}$

(c) Age $<10,>40$

(d) $>7$ days between jaundice and coma

(e) Drug Toxicity. 
Transfer to a center capable of performing liver transplantation is mandatory if phase $2-3$ poisoning with evidence of liver failure is present.

Escudié et al. reported on 27 patients admitted for amanita poisoning in a 2006 article published in the Journal of Hepatology. The aim of the study was to reevaluate the established criteria for liver transplant status post amatoxin poisoning. $30 \%$ of the patients analyzed died due to ingestion. An early predictor of fatal outcome was the onset of diarrhea less than eight hours after ingestion, as the accuracy of this indicator was found to be $78 \%$. The study also showed that the King's College criteria were superior to Clichy's and Ganzert's criteria (accuracy of 100\% compared to $85 \%$ and $85 \%$, resp.). Another interesting observation was that the development of renal impairment and encephalopathy was not accurate predictors of fatal amatoxin ingestion. Finally, it was observed that a prothrombin index below $10 \%$ for four days or more after ingestion had 100\% accuracy for predicting a fatal outcome. As a result of the study, the writers concluded that liver transplant should be advocated for early in patients with onset of diarrhea less than eight hours after ingestion and in patients with a prothrombin index lower than $10 \%$ four or more days after ingestion [13].

The most important predictor of survival in fulminant liver failure is whether or not a patient receives a liver transplantation. The use of hepatocyte-based bioartificial liver (BAL) has recently emerged as a promising bridge from the acute phase of fulminant liver failure to ultimate liver transplant. This therapy allows for plasmapheresis of a patient's blood via a femoral vein catheter and subsequent filtering through porcine hepatocytes. BAL was described and studied extensively by Demetriou et al. and their outcomes using BAL revealed that the group of patients in fulminant and subfulminant liver failure had a $44 \%$ statistically significant reduction in mortality rate. Although the full mechanism of BAL is still not completely understood, it has been identified as a suitable bridge therapy for patients with fulminant liver failure awaiting transplant [14].

\section{Conclusions}

Amatoxin is an often fatal toxin when ingested. There is no specific therapy for poisoning, but subsequent to poisoning, multidose-activated charcoal is recommended as well as careful correction and maintenance of electrolytes, coagulation factors, and fluids. Consideration for use of steroids, mannitol, and penicillin should be considered as well. Transfer to a tertiary care facility may be warranted for those with hepatic failure and a potential need for transplantation.

\section{References}

[1] P. Poucheret, F. Fons, J. C. Doré, D. Michelot, and S. Rapior, "Amatoxin poisoning treatment decision-making: pharmacotherapeutic clinical strategy assessment using multidimensional multivariate statistic analysis," Toxicon, vol. 55, no. 7, pp. 1338-1345, 2010.
[2] D. R. Benjamin, Mushrooms: Poisons and Panaceas-A Handbook for Naturalists, Mycologists and Physicians, W.H. Freeman, 1995.

[3] E. G. Yamada, J. Mohle-Boetani, K. R. Olson, and S. B. Werner, "Mushroom poisoning due to amatoxin. Northern California, Winter 1996-1997," Western Journal of Medicine, vol. 169, no. 6, pp. 380-384, 1998.

[4] P. Zhou, J. Xia, G. Guo et al., "A Macaca mulatta model of fulminant hepatic failure," World Journal of Gastroenterology, vol. 18, no. 5, pp. 435-444, 2012.

[5] J. H. Diaz, "Syndromic diagnosis and management of confirmed mushroom poisonings," Critical Care Medicine, vol. 33, no. 2, pp. 427-436, 2005.

[6] A. Meixner, "Amatoxin-Nachweis in Pilzen," Zeitschrift fur Mykologie, vol. 45, pp. 137-140, 1979.

[7] J. A. Beutler and P. P. Vergeer, "Amatoxins in American mushrooms: evaluation of the Meixner test," Mycologia, vol. 72, pp. 1142-1144, 1980.

[8] G. L. Floersheim, "Treatment of human amatoxin mushroom poisoning: myths and advances in therapy," Medical Toxicology and Adverse Drug Experience, vol. 2, no. 1, pp. 1-9, 1987.

[9] R. Gažák, D. Walterová, and V. Křen, "Silybin and silymarinnew and emerging applications in medicine," Current Medicinal Chemistry, vol. 14, no. 3, pp. 315-338, 2007.

[10] T. C. Tong, M. Hernandez, W. H. Richardson et al., "Comparative treatment of alpha-amanitin poisoning with $\mathrm{N}$ acetylcysteine, benzylpenicillin, cimetidine, thioctic acid, and silybin in a murine model," Annals of Emergency Medicine, vol. 50, no. 3, pp. 282-288, 2007.

[11] F. Enjalbert, S. Rapior, J. Nouguier-Soulé, S. Guillon, N. Amouroux, and C. Cabot, "Treatment of amatoxin poisoning: 20-Year retrospective analysis," Journal of Toxicology, vol. 40, no. 6, pp. 715-757, 2002.

[12] L. Giannini, A. Vannacci, A. Missanelli et al., "Amatoxin poisoning: a 15-year retrospective analysis and follow-up evaluation of 105 patients," Clinical Toxicology, vol. 45, no. 5, pp. 539-542, 2007.

[13] L. Escudié, C. Francoz, J. P. Vinel et al., "Amanita phalloides poisoning: reassessment of prognostic factors and indications for emergency liver transplantation," Journal of Hepatology, vol. 46, no. 3, pp. 466-473, 2007.

[14] A. A. Demetriou, R. S. Brown, R. W. Busuttil et al., "Prospective, randomized, multicenter, controlled trial of a bioartificial liver in treating acute liver failure," Annals of Surgery, vol. 239, no. 5, pp. 660-670, 2004. 


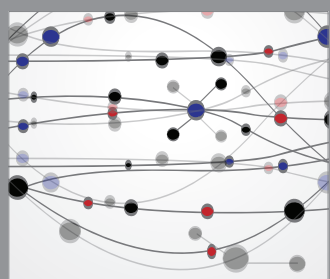

The Scientific World Journal
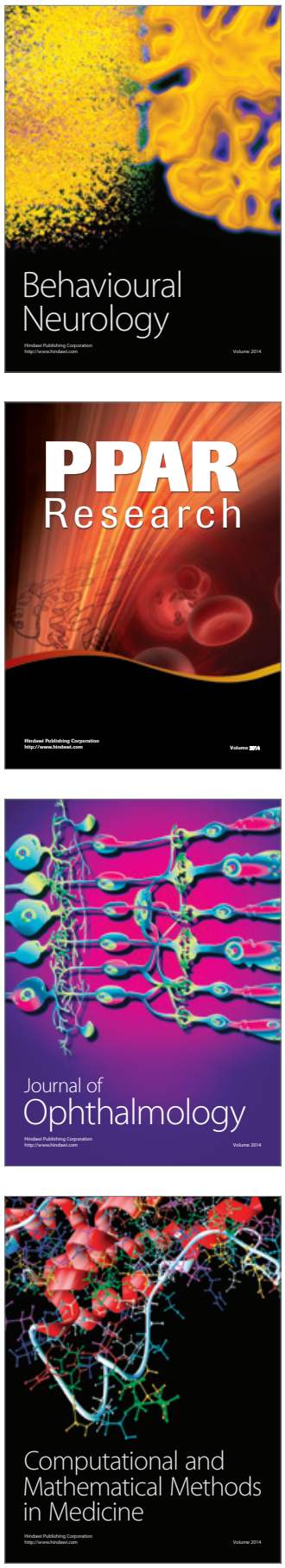

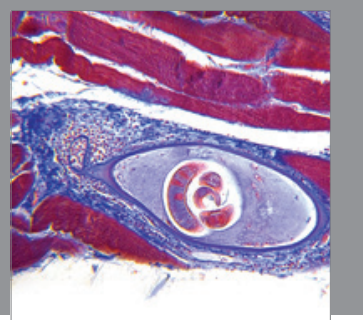

Gastroenterology

Research and Practice
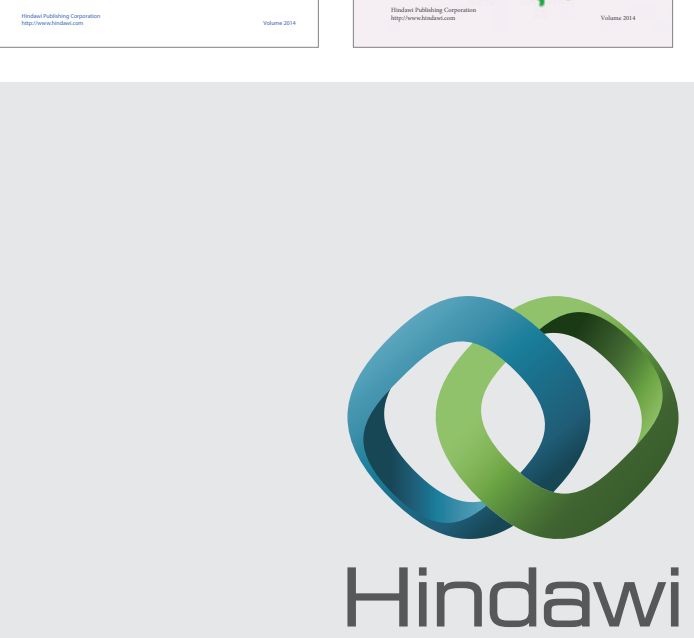

Submit your manuscripts at

http://www.hindawi.com
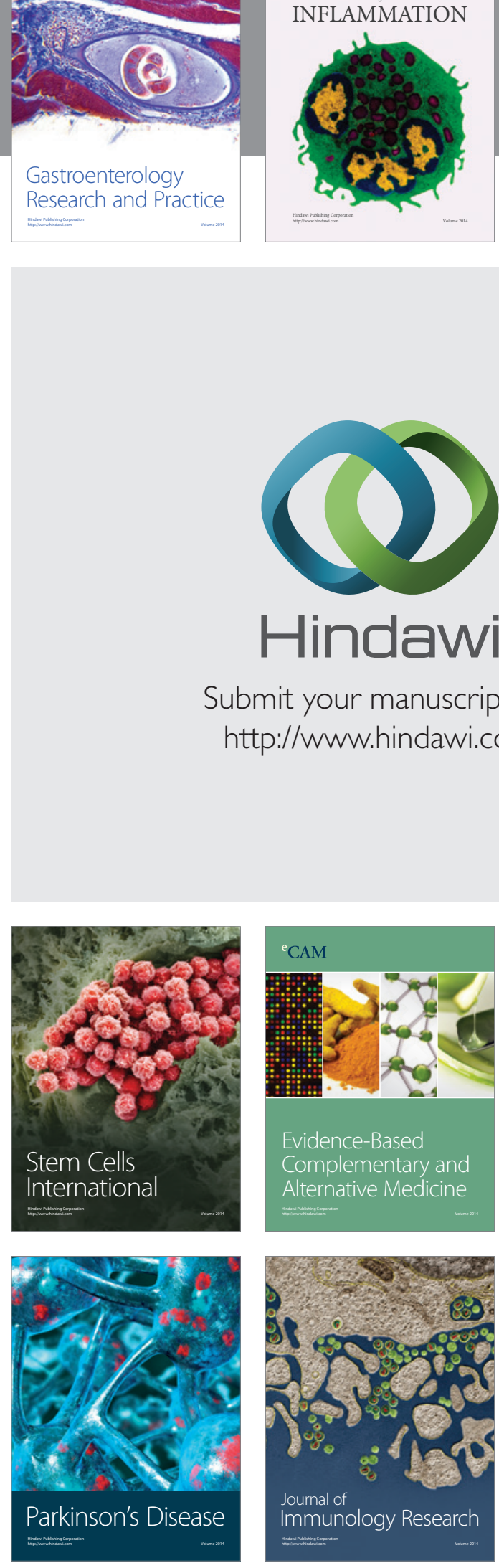

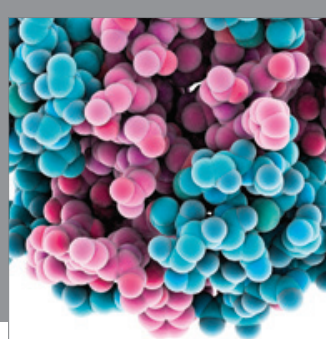

Diabetes Research
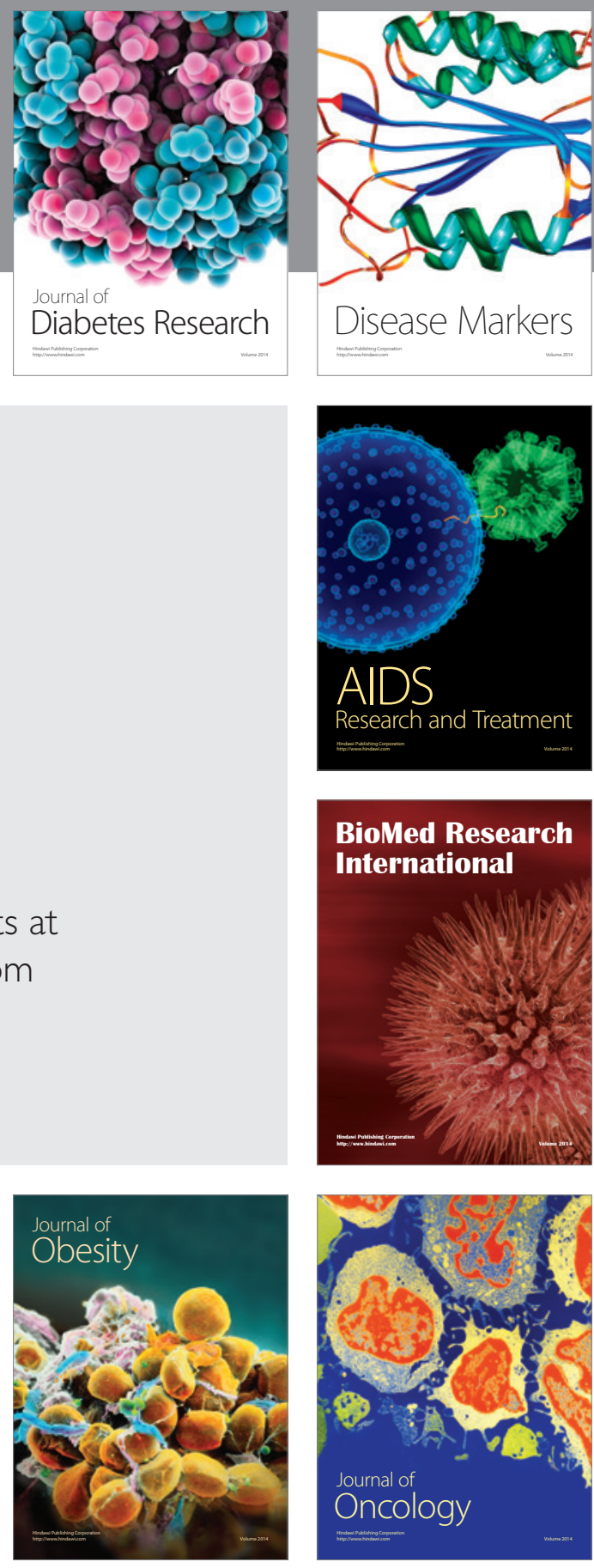

Disease Markers

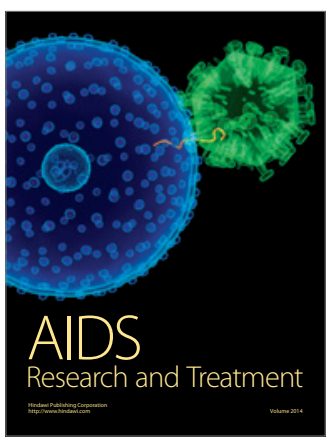

BioMed Research

International
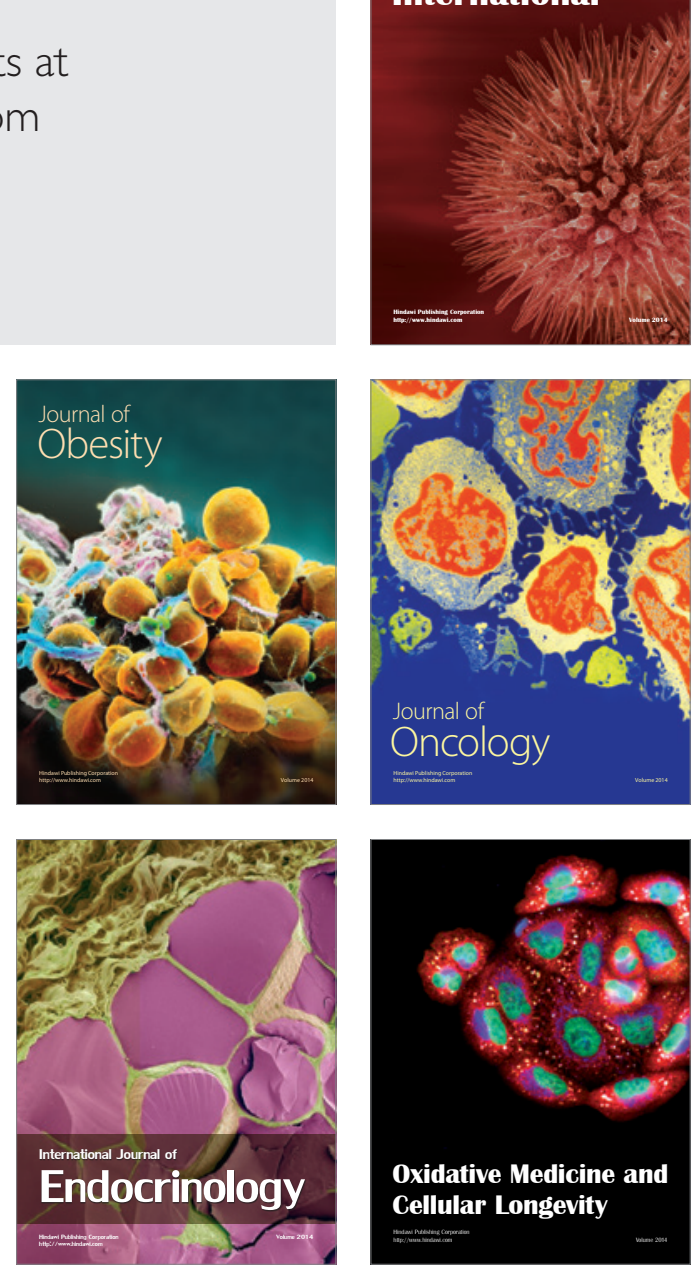